\title{
Cathodoluminescence Microscopies of Color Centers in Bulk and 2D Materials
}

\author{
Ben Lawrie $^{1}$, Matthew Feldman ${ }^{2}$ and Jordan Hachtel ${ }^{1}$ \\ ${ }^{1}$ Oak Ridge National Laboratory, Oak Ridge, TN, Tennessee, United States, ${ }^{2}$ Vanderbilt University, Oak \\ Ridge, Tennessee, United States
}

Cathodoluminescence (CL) microscopy increasingly provides a unique tool for sub-diffraction-limited characterization of color centers and excitons in nanoscale and 2D materials [1,2] and for the characterization of plasmonic and nanophotonic systems [3,4]. However, despite the widespread availability of converged electron beam-probes, free carrier diffusion prior to radiative recombination limits CL spatial resolution. Because of this free carrier diffusion, attempts to excite individual defects have typically resulted in the excitation of ensembles of defects such that the collected CL signal exhibits photon bunching instead of photon antibunching [1]. Cathodoluminescence measurements in a scanning transmission electron microscope have successfully addressed individual defect centers [2], but the same effect has not yet been reported for CL acquired in a scanning electron microscope (SEM).

Here, we will explore the use of nanopatterning, localized strain induced through electron-beam induced deposition, temperature tuning, and plasmonic near-field control in order to characterize and control color centers in diamond and 2D materials. In particular, we will describe electron-beam induced deposition of gold nanoplasmonic structures around color centers with in-situ CL characterization in order to optimize the radiative recombination rates of individual color centers. Further, we will explore the electron beam annealing of these nanoplasmonic structures as a mechanism for controlling the local strain environment around color centers, enabling dynamic control of their optical properties. We will describe temperature tuning from $8-300 \mathrm{~K}$ as a means for controlling free-carrier diffusion and correspondingly the photon statistics of electron-beam driven color centers. Finally, we will compare a range of single photon detectors in order to optimize the detector design for CL microscopy. Superconducting nanowire single photon detectors provide a low-dark count, high quantum efficiency platform ideal for multidimensional spectroscopies with low photon fluences, but their small active areas limit the SEM-CL field of view. We will explore the suitability of these detectors and more conventional avalanche photodiodes for specific material systems.

\section{References}

[1] Feldman et al. "Colossal photon bunching in quasiparticle-mediated nanodiamond cathodoluminescence." Physical Review B 97, 081494 (2018)

[2] Bourrellier et al. "Bright single photon emission at point defects in h-BN" Nano Letters 16, 4317 (2016)

[3] Hachtel et al., "Spatially and spectrally resolved orbital angular momentum interactions in plasmonic vortex generators" Light: Science \& Applications, 8, 1 (2019)

[4] Hachtel et al., "Polarization- and wavelength-resolved near-field imaging of complex plasmonic modes in Archimedean nanospirals" Optics Letters 43, 927 (2018) 